\title{
Apolipoprotein E gene polymorphism and renal function are associated with apolipoprotein E concentration in patients with chronic kidney disease
}

\author{
Monika Czaplińska ${ }^{1 *} \mathbb{D}$, Agnieszka Ćwiklińska², Monika Sakowicz-Burkiewicz³, Ewa Wieczorek², Agnieszka Kuchta²,
} Robert Kowalski ${ }^{4}$, Barbara Kortas-Stempak², Alicja Dębska-Ślizień ${ }^{1}$, Maciej Jankowski and Ewa Król ${ }^{1}$

\begin{abstract}
Background: Chronic kidney disease (CKD) associates with complex lipoprotein disturbances resulting in high cardiovascular risk. Apolipoprotein E (APOE) is a polymorphic protein with three common isoforms (E2; E3; E4) that plays a crucial role in lipoprotein metabolism, including hepatic clearance of chylomicrons and very low-density lipoprotein (VLDL) remnants, and reverse cholesterol transport. It demonstrates anti-atherogenic properties but data concerning the link between polymorphism and level of APOE in CKD patients are inconclusive.

The aim of our research was to assess the relationship between APOE gene polymorphism and APOE concentration and its redistribution among lipoproteins along with CKD progression.

Methods: 90 non-dialysed CKD patients were included into the study. Real time PCR was used for APOE genotyping. APOE level was measured in serum and in isolated lipoprotein fractions (VLDL; IDL + HDL; HDL). Kidney function was assessed using eGFR CKD-EPI formula.

Results: The population was divided into three APOE genotype subgroups: $E 2(\varepsilon 2 \varepsilon 3), E 3(\varepsilon 3 \varepsilon 3)$ and $E 4(\varepsilon 3 \varepsilon 4)$. The highest APOE level was observed for the E2 subgroup $(p<0.001)$. APOE concentration positively correlated with eGFR value in the E2 subgroup ( $r=0.7, p<0.001)$ but inversely in the E3 subgroup $(r=-0.29, p=0.02)$.). A lower concentration of APOE in the E2 subgroup was associated with its diminished contents in HDL and IDL + LDL particles. In the E3 subgroup, the higher concentration of APOE was related to the increased number of non-HDL lipoproteins.
\end{abstract}

Conclusion: In patients with CKD, APOE genotype as well as renal function are associated with the concentration of APOE and its redistribution among lipoprotein classes.

Keywords: Chronic kidney disease, Apolipoprotein E, Apolipoprotein E gene polymorphism, Lipoprotein

\section{Background}

Chronic kidney disease (CKD) is one of the current leading public health problems due to increasing frequency, complications as well as high mortality resulting from accelerated atherosclerosis [1]. The main cause of death in this population is cardiovascular disease (CVD) connected with dyslipidaemia, which is observed at early

\footnotetext{
* Correspondence: cackowska@gumed.edu.pl

${ }^{1}$ Clinic \& Chair of Nephrology, Transplantology and Internal Medicine,

Medical University of Gdańsk, Dębinki 7, 80-211 Gdańsk, Poland

Full list of author information is available at the end of the article
}

stages of renal failure and associated with the degree of glomerular filtration rate declining [2, 3]. Hypertriglyceridemia, accumulation of intact or partially metabolised apolipoprotein B (APOB)-containing lipoproteins (very low-density lipoprotein (VLDL), intermediate density lipoprotein (IDL), low density lipoprotein (LDL)), and reduced concentration of high density lipoprotein (HDL) are well documented lipid disturbances in CKD [3-7]. Other studies have also shown that the concentration and distribution of apolipoproteins - which play a crucial role in lipid metabolism - are also disturbed in CKD [8].

(c) The Author(s). 2019 Open Access This article is distributed under the terms of the Creative Commons Attribution 4.0 International License (http://creativecommons.org/licenses/by/4.0/), which permits unrestricted use, distribution, and 
Apolipoprotein E (APOE), a $34 \mathrm{kDa}$ glycoprotein, is produced mainly in the liver but its local production has been documented in the brain, kidneys, spleen, adrenals and macrophages [9]. It is a component of all classes of lipoproteins except small dense LDL. It circulates between HDL and APOB-containing lipoproteins in plasma [10, 11]. APOE acts as a ligand for receptor-mediated clearance of chylomicrons (CM) and VLDL remnants from the circulation to the liver and takes part in reverse cholesterol transport (RCT) as a component of HDL [11]. The protective role of APOE in atherosclerosis development was first proven in animal models [12]. In further research it was demonstrated that APOE is an anti-atherogenic protein also in humans $[9,13,14]$. It was also shown that cholesterol-loaded macrophages demonstrated higher APOE gene expression; APOE deficiency in these cells decreased cholesterol efflux and led to atherosclerosis plaques formation [15].

There are three common APOE isoforms (APOE2, APOE3, APOE4) coded by three alleles $(\varepsilon 2, \varepsilon 3, \varepsilon 4)$ of the $A P O E$ gene on chromosome $19 \mathrm{q} 13.2$, therefore there are six $A P O E$ gene polymorphisms: $A P O E 2 / 2, A P O E 3 / 3$, $A P O E 4 / 4, A P O E 2 / 3, A P O E 3 / 4$ and APOE2/4 [11, 16]. The isoforms differ in two amino acids at residues 112 and 158 - APOE2 has cysteine at both sides, APOE4 has arginines at both sides and APOE3 has cysteine at 112 residue and arginine at 158 residue [11]. Due to the differences in APOE isoforms structure, they possess diverse binding capacity to receptors as well as lipoprotein-binding preferences that results in a various influence on the lipoproteins metabolism [11]. APOE3 and APOE4 isoforms bind to the LDL receptor with similar potent affinity, whereas APOE2 is defective in interaction with the receptor - only $2 \%$ of normal activity [16]. APOE2 and APOE3 isoforms preferentially bind to small HDL, while APOE4 to large triglyceride (TG)-rich VLDL particles [17]. APOE3 is the most common isoform (around $77 \%$ of the Caucasian population) and does not impair lipoprotein metabolism. APOE4 occurs in $15 \%$ of the population and can lead to elevated LDL-C concentration. APOE2 is the rarest isoform (8\%) and might be related with hypertriglyceridemia $[17,18]$. These disturbances in various ways contribute to accelerated atherosclerosis. The APOE gene polymorphism also influences APOE concentration in serum; the highest levels were measured in $\varepsilon 2$ allele carriers, the lowest in $\varepsilon 4$ carriers [18].

Although APOE has anti-atherogenic features, there are ambiguous data about the link between APOE concentration and CVD risk [19-21]. Surprisingly, it has been shown in many studies that CVD patients had elevated APOE concentration [18, 19, 22-24]. However, researchers have highlighted that APOE distribution between lipoproteins has a crucial meaning and total
APOE concentration in serum is not an appropriate parameter for the evaluation of CVD risk. It has been proven that increased APOE level in the CVD population resulted from higher APOE content in APOB-containing lipoproteins and that factor could be a proper marker for this purpose [22, 25].

The important role of APOE in lipid metabolism and CVD development in CKD patients has also been emphasised by many researchers [22, 26, 27]. The polymorphism of the $A P O E$ gene has an influence on APOE concentration as well as lipids and lipoproteins disturbances. Wang $\mathrm{Y}$ et al. proved that hemodialysed $\varepsilon 4$ allele carriers have higher total cholesterol (TC), TG, and LDL-C [27]. $\varepsilon 2$ allele carriers develop accumulation of cholesterol-rich VLDL [28]. However, there is poor knowledge about the relationship between $A P O E$ gene polymorphism, APOE level, its distribution among lipoproteins and the degree of kidney dysfunction, especially at early stages of CKD. These findings could shed new light on the pathomechanism of lipid-related disorders and accelerated atherosclerosis in CKD patients.

The aim of our research was to assess the relationship between $A P O E$ gene polymorphism and APOE serum concentration and its redistribution among lipoprotein classes along with CKD progression.

\section{Materials and methods \\ Study group}

Ninety non-dialyzed adult CKD patients at the stage from 3a to 4 (eGFR $59-16 \mathrm{ml} / \mathrm{min} / 1.73 \mathrm{~m}^{2}$ ) being under the care of the Outpatient Nephrology Clinic of the University Clinic Centre in Gdańsk (Poland) were included into the study. The exclusion criteria included: diabetes, liver diseases, infections, nephrotic proteinuria, active neoplasm, immunosuppressive treatment including steroids, heparin treatment due to lipoprotein lipase activation and hypolipidemic agents except statins.

The Independent Bioethics Commission for Research of the Medical University of Gdańsk (Poland) approved the study, performed in accordance with the Declaration of Helsinki and all the participants provided written informed consent.

Demographic data about medications, traditional risk factors of CVD (age, sex, smoking cigarettes, hypertension, obesity) and personal medical histories were collected during a medical appointment. During the physical examination 3-times blood pressure (BP) in a few minute intervals were measured with an Omron Upper Arm Blood Pressure Monitor. The first measurement was ignored and the mean value was calculated from the second and third measurements. Hypertension was defined as a systolic $\mathrm{BP} \geq 140 \mathrm{mmHg}$ and/or diastolic $\mathrm{BP} \geq 90 \mathrm{mmHg}$ in two medical appointments in the Outpatient Clinic and/or current antihypertensive 
treatment. Moreover, body mass index (BMI) and waist-to-hip ratio (WHR) were assessed. Obesity was diagnosed on the basis of a BMI $\geq 30 \mathrm{~kg} / \mathrm{m}^{2}$.

\section{Methodology}

Blood samples were obtained from the vein after an overnight fasting of a minimum of ten hours. VLDL was isolated from blood serum by ultracentrifugation [7, 29]. HDL was isolated by precipitation of APOB-containing lipoproteins with heparin and manganese chloride from the ultracentrifugal $\mathrm{d}>1.006 \mathrm{~g} / \mathrm{ml}$ fraction [30]. In the serum and in isolated VLDL and HDL fractions the concentration of lipids and apolipoproteins were measured. (IDL + LDL)-lipid and apolipoprotein levels were calculated as a difference: total concentration of component in serum VLDL - HDL.

Serum total protein (TP), albumin, TC, TG and plasma glucose were measured using commercially available kits obtained from Pointe Scientific (Warsaw, Poland). Apolipoprotein $\mathrm{AI}, \mathrm{E}$ and $\mathrm{B}$ were measured by immunonephelometry using kits obtained from Siemens Healthcare Diagnostics (Erlangen, Germany) [31, 32]. LDL-C was calculated using Friedewald formula.

Serum creatinine was measured using the enzymatic method that has calibration traceable to an IDMS reference measurement procedure (Abbott Diagnostics Inc., Santa Clara, CA, USA) [33]. CKD-EPI equation was used to estimate GFR.

Genomic DNA was isolated by manual extraction using columns from a commercially available kit obtained from A\&A Biotechnology (Gdynia, Poland).

$A P O E$ genotyping (rs429358, rs7412) was performed by real time PCR reaction using MutaREAL ${ }^{\circ}$ APOE real time PCR Kit obtained from Immundiagnostik AG (Bensheim, Germany) [34]. Briefly, for analysis of $A P O E$ genotyping (rs429358 or rs7412) the potentially mutated region of the $A P O E$ gene was amplified by PCR in a capillary by LightCycler (Roche, Basel, Switzerland) using genomic DNA. Amplification products were analyzed afterwards in a melting-point curve analysis with mutation specific hybridization probes detected at $670 \mathrm{~nm}$. The melting-point curve analysis allowed a clear identification of wildtype, heterozygous or homozygous genotypes. For rs429358, rs7412 APOE genotyping the Hybridization Probes were designed in this way that their sequence fits exactly onto the CGC-sequence. Therefore, in the melting point curve analysis the mutation peak arose earlier in the presence of TGC-sequence because of the introduced mismatch (= sequences are not $100 \%$ homologous). In the case of heterozygous genotype two peaks were generated - one with a lower temperature (TGC) and one with a higher temperature (CGC).

\section{Statistical analysis}

Continuous variables were expressed as a mean \pm standard deviation (SD) or as a median and 10th - 90th percentiles, and were compared using the unpaired Student's t-test, Mann-Whitney U test, or one-way analysis of variance, as appropriate. Categorical variables, expressed as a number (percent), were compared using $\mathrm{X}^{2}$ test. Correlations were estimated using Spearman's rank method. Statistical analyses were performed using STATISTICA PL 12.0 (Statsoft, Cracow, Poland). P value of $<0.05$ was considered statistically significant.

\section{Results}

\section{Characteristics of the study group}

The baseline characteristics of the CKD patients are presented in Table 1 . The study population was categorised into 3 APOE subgroups according to $A P O E$ gene polymorphism: subgroup E2 ( $\varepsilon 2 \varepsilon 3$ allele carriers), E3 ( $\varepsilon 3 \varepsilon 3)$, and $\mathrm{E} 4(\varepsilon 3 \varepsilon 4)$. In our research group there were neither homozygotes APOE2-2 and APOE4-4 nor APOE2-4 heterozygotes. The analyzed subgroups did not differ in their age, sex, BMI index, status of smoking cigarettes, statin treatment, as well as kidney function measured by eGFR CKD-EPI value (Table 1). They also did not differ in their TP, albumin and glucose levels (Table 1).

There were no significant differences in a basic lipid profile parameters as well as APOB and APOAI levels between the APOE subgroups (Table 2).

\section{Concentration of APOE in serum and in lipoprotein fractions according to $A P O E$ genotype}

There were statistically significant differences in total APOE and APOE-HDL concentrations as well as in APOE-HDL/ APOAI ratio depending on APOE genotype (Table 3). In the E2 subgroup, the total APOE and APOE-HDL levels were significantly higher in comparison to the E3 (for total APOE: $p<0.05$; APOE-HDL: $p<0.001$; APOE-HDL/ APOAI: $p<0.001$ ) and E4 (for total APOE: $p=0.002$; APOE-HDL: $p<0.001$; APOE-HDL/APOAI: $p<0.001)$ subgroups (Table 3). There were no differences in the APOE-non-HDL, APOE-IDL + LDL and APOE-VLDL concentrations nor in the APOE/APOB ratios between the subgroups (Table 3).

\section{Relationship between eGFR CKD-EPI and APOE concentration in serum and in lipoprotein fractions according to $A P O E$ genotype}

In the entire study group there was no correlation between APOE serum concentration and eGFR value (Fig. 1a, Table 4).

For the E2 subgroup, APOE serum concentration was positively correlated with eGFR value (Fig. 1b, Table 4); this relationship remained statistically significant in multiple linear regression after adjusting for sex, BMI, and statin 
Table 1 Baseline characteristic of the study groups

\begin{tabular}{|c|c|c|c|c|c|}
\hline \multirow[t]{2}{*}{ Parameter } & \multirow[t]{2}{*}{ Total } & \multicolumn{3}{|l|}{ APOE subgroup } & \multirow{2}{*}{$\begin{array}{l}P \\
\text { value }\end{array}$} \\
\hline & & E2 & E3 & E4 & \\
\hline cases & $90(100 \%)$ & $18(20 \%)$ & $58(64 \%)$ & $14(16 \%)$ & NA \\
\hline $\operatorname{sex}[\mathrm{F} / \mathrm{M}]$ & $37 / 53$ & $8 / 10$ & $23 / 35$ & $6 / 8$ & $0.92^{* *}$ \\
\hline age [years] & $68 \pm 10$ & $65 \pm 12$ & $68 \pm 11$ & $66 \pm 10$ & $0.50^{*}$ \\
\hline hypertension & $73(81 \%)$ & $17(94 \%)$ & $45(78 \%)$ & $11(79 \%)$ & $0.19^{* *}$ \\
\hline obesity & $27(30 \%)$ & $7(39 \%)$ & $15(26 \%)$ & $5(36 \%)$ & $0.50^{* *}$ \\
\hline BMI $\left[\mathrm{kg} / \mathrm{m}^{2}\right]$ & $28 \pm 5$ & $29 \pm 7$ & $27 \pm 4$ & $29 \pm 5$ & $0.27^{*}$ \\
\hline elevated WHR ( $\geq 1$ for males, $\geq 0.85$ for females) & $45(50 \%)$ & $11(61 \%)$ & $26(45 \%)$ & $8(57 \%)$ & $0.58^{* *}$ \\
\hline smoking cigarettes & $40(44 \%)$ & $11(61 \%)$ & $23(40 \%)$ & $6(42 \%)$ & $0.28^{* *}$ \\
\hline statin therapy & $48(53 \%)$ & $11(61 \%)$ & $27(47 \%)$ & $10(71 \%)$ & $0.18^{* *}$ \\
\hline G3a stage CKD & $28(31 \%)$ & $4(22 \%)$ & $20(35 \%)$ & $4(29 \%)$ & $0.59^{* *}$ \\
\hline G3b stage CKD & $42(47 \%)$ & $7(39 \%)$ & $29(50 \%)$ & $6(42 \%)$ & $0.68^{* *}$ \\
\hline G4 stage CKD & $20(22 \%)$ & $7(39 \%)$ & $9(15 \%)$ & $4(29 \%)$ & $0.11^{* *}$ \\
\hline creatinine $[\mathrm{mg} / \mathrm{dl}]$ & $1.6(1.2-2.6)$ & $1.7(1.0-2.8)$ & $1.6(1.1-2.2)$ & $1.6(1.3-2.9)$ & $0.24^{* * *}$ \\
\hline eGFR CKD-EPI $\left[\mathrm{ml} / \mathrm{min} / 1.73 \mathrm{~m}^{2}\right]$ & $39 \pm 12$ & $37 \pm 13$ & $41 \pm 12$ & $36 \pm 13$ & $0.34^{*}$ \\
\hline total protein $[\mathrm{g} / \mathrm{l}]$ & $64.1 \pm 4.1$ & $64.5 \pm 4.3$ & $63.7 \pm 4.0$ & $64.3 \pm 4.5$ & $0.49^{*}$ \\
\hline albumin [g/l] & $42.9 \pm 3.1$ & $42.6 \pm 3.3$ & $43.0 \pm 3.1$ & $42.9 \pm 2.7$ & $0.90^{*}$ \\
\hline glucose $[\mathrm{mg} / \mathrm{dl}]$ & $95.0(82.8-109.8)$ & $95.3(85.7-107)$ & $94.4(81.7-108.7)$ & $96.7(83-121)$ & $0.86^{* * *}$ \\
\hline
\end{tabular}

Continuous values are presented as mean \pm standard deviation (SD) or median and range (10th and 90th percentiles). Differences among the subgroups were analysed using ANOVA*, Pearson's chi-squared test**, or Kruskall-Wallis test***

BMI - body mass index, WHR - waist-to-hip ratio, CKD - chronic kidney disease, eGFR CKD-EPI - estimated glomerular filtration rate-chronic kidney disease-epidemiology collaboration

Subgroups: E2: $\varepsilon 2 \varepsilon 3$ subjects; E3: $\varepsilon 3 \varepsilon 3$ subjects; E4: $\varepsilon 3 \varepsilon 4$ subjects

therapy $(\beta=0.806$; standard error $(\mathrm{SE})=0.184 ; p<0.001)$. For the E3 subgroup, the APOE level was inversely correlated with eGFR value (Fig. 1c, Table 4) and this relationship also remained significant after adjustment for the above-mentioned factors $(\beta=-0.286$; $\mathrm{SE}=0.122 ; p=0.02)$.

For the E2 subgroup there was lower APOE-HDL and APOAI concentrations, and APOE-HDL/APOAI ratio, along with a decline in eGFR (Fig. 2, Table 4). The same relationship was observed for APOE level and APOE/ APOB ratio in IDL + LDL (Fig. 2, Table 4). For VLDL there was an opposite tendency; APOE-VLDL level negatively correlated with eGFR value(Table 4).
For the E3 subgroup, there was no relationship between APOE-HDL level and eGFR. Conversely, in non-HDL lipoproteins, the APOE and APOB concentrations increased along with eGFR decline, and the APOE/ APOB ratio remained constant (Table 4).

For the E4 subgroup, we did not observe a statistically significant relationship between total APOE level in serum or individual lipoprotein fractions, and eGFR (Table 4).

After division of the APOE subgroups according to CKD stage, we observed statistically significant differences in APOE concentration (Fig. 3). In the G3a CKD stage

Table 2 Lipid parameters in the study groups

\begin{tabular}{|c|c|c|c|c|c|}
\hline \multirow[t]{2}{*}{ Parameter } & \multirow[t]{2}{*}{ Total } & \multicolumn{3}{|c|}{ APOE subgroup } & \multirow{2}{*}{$\begin{array}{l}P \\
\text { value }\end{array}$} \\
\hline & & E2 & E3 & E4 & \\
\hline $\mathrm{TC}[\mathrm{mg} / \mathrm{dl}]$ & $204 \pm 48$ & $201 \pm 26$ & $207 \pm 54$ & $198 \pm 42$ & $0.77^{*}$ \\
\hline Non-HDL-C [mg/dl] & $154 \pm 47$ & $148 \pm 26$ & $156 \pm 53$ & $154 \pm 43$ & $0.65^{*}$ \\
\hline LDL-C [mg/dl] & $130 \pm 44$ & $121 \pm 22$ & $134 \pm 50$ & $125 \pm 39$ & $0.29^{*}$ \\
\hline $\mathrm{HDL}-\mathrm{C}[\mathrm{mg} / \mathrm{dl}]$ & $50 \pm 13$ & $54 \pm 15$ & $50 \pm 12$ & $43 \pm 10$ & $0.31^{*}$ \\
\hline $\mathrm{TG}[\mathrm{mg} / \mathrm{dl}]$ & $112(64-182)$ & $122(64-241)$ & $98(63-164)$ & $121(69-277)$ & $0.21^{* *}$ \\
\hline
\end{tabular}

Values are presented as mean \pm SD or median and range (10th and 90th percentiles). Differences among the subgroups were analysed using ANOVA* or Kruskall-Wallis test**

TC - total cholesterol, LDL-C - low density lipoprotein-cholesterol, HDL-C - high density lipoprotein-cholesterol, TG - triglycerides, APOB - apolipoprotein B, APOAI - apolipoprotein Al, non-HDL-C - non-high density lipoprotein-cholesterol (very low-density-cholesterol + intermediate density lipoprotein-cholesterol + LDL-C) Subgroups: E2: $\varepsilon 2 \varepsilon 3$ subjects; E3: $\varepsilon 3 \varepsilon 3$ subjects; E4: $\varepsilon 3 \varepsilon 4$ subjects 
Table 3 APOE, APOAl, and APOB concentrations $[\mathrm{mg} / \mathrm{dl}]$ in serum and in lipoprotein fractions in the study groups

\begin{tabular}{|c|c|c|c|c|c|}
\hline \multirow[t]{2}{*}{ Parameter } & \multirow[t]{2}{*}{ Total } & \multicolumn{3}{|l|}{ APOE subgroup } & \multirow[t]{2}{*}{$P$ value } \\
\hline & & $\mathrm{E} 2$ & E3 & E4 & \\
\hline APOE & $4.08 \pm 1.27$ & $4.94 \pm 1.47$ & $4.00 \pm 1.15$ & $3.34 \pm 0.80$ & $<0.001^{*}$ \\
\hline APOE-HDL & $1.45 \pm 0.60$ & $2.08 \pm 1.15$ & $1.38 \pm 0.50$ & $0.90 \pm 0.34$ & $<0.001^{* *}$ \\
\hline APOE-non-HDL & $2.66 \pm 0.93$ & $2.86 \pm 1.15$ & $2.64 \pm 0.92$ & $2.44 \pm 0.66$ & 0.43 \\
\hline APOE-IDL + LDL & $2.06 \pm 0.85$ & $2.23 \pm 1.25$ & $2.09 \pm 0.72$ & $1.70 \pm 0.62$ & 0.23 \\
\hline APOE-VLDL & $0.57 \pm 0.42$ & $0.63 \pm 0.52$ & $0.51 \pm 0.37$ & $0.70 \pm 0.44$ & 0.23 \\
\hline APOAI & $168 \pm 30$ & $178 \pm 33$ & $166 \pm 30$ & $164 \pm 25$ & 0.49 \\
\hline APOE-HDL/APOAI & $0.0086 \pm 0.0031$ & $0.0117 \pm 0.0019$ & $0.0082 \pm 0.0027$ & $0.0056 \pm 0.0023$ & $<0.0001^{* * *}$ \\
\hline APOB & $94 \pm 25$ & $91 \pm 11$ & $95 \pm 27$ & $97 \pm 30$ & 0.95 \\
\hline APOB-IDL + LDL & $85 \pm 24$ & $81 \pm 12$ & $85 \pm 25$ & $87 \pm 37$ & 0.97 \\
\hline APOB-VLDL & $8.8 \pm 4.2$ & $9.4 \pm 4.2$ & $8.1 \pm 4.0$ & $10.7 \pm 4.4$ & 0.08 \\
\hline APOE/APOB in non-HDL & $0.029 \pm 0.0085$ & $0.032 \pm 0.012$ & $0.029 \pm 0.0074$ & $0.026 \pm 0.0068$ & 0.40 \\
\hline APOE/APOB in IDL + LDL & $0.025 \pm 0.010$ & $0.028 \pm 0.015$ & $0.025 \pm 0.0082$ & $0.021 \pm 0.0073$ & 0.15 \\
\hline APOE/APOB in VLDL & $0.071 \pm 0.044$ & $0.070 \pm 0.046$ & $0.071 \pm 0.044$ & $0.073 \pm 0.045$ & 0.95 \\
\hline
\end{tabular}

Values are presented as mean \pm SD. Differences among the subgroups were analysed using ANOVA and test post hoc Tukey's test

APOE - apolipoprotein E, APOAI - apolipoprotein Al, APOB - apolipoprotein B, non-HDL - non-high density lipoprotein (VLDL + IDL + LDL), HDL - high density lipoprotein, VLDL - very low-density lipoprotein, IDL - intermediate density lipoprotein, LDL - low density lipoprotein

Subgroups: E2: $\varepsilon 2 \varepsilon 3$ subjects; E3: $\varepsilon 3 \varepsilon 3$ subjects; E4: $\varepsilon 3 \varepsilon 4$ subjects

${ }^{*} \mathrm{E} 2$ vs E4 $p=0.002$, E2 vs E3 $p=0.052$, E3 vs E4 $p=0.291$, ** E2 vs E4 $p<0.001$, E2 vs E3 $\mathrm{p}<0.001, \mathrm{E} 3$ vs E4 $p=0.039$, *** E2 vs E4 p<0.001, E2 vs E3 p<0.001, E3 vs $\mathrm{E} 4 \mathrm{p}=0.016$

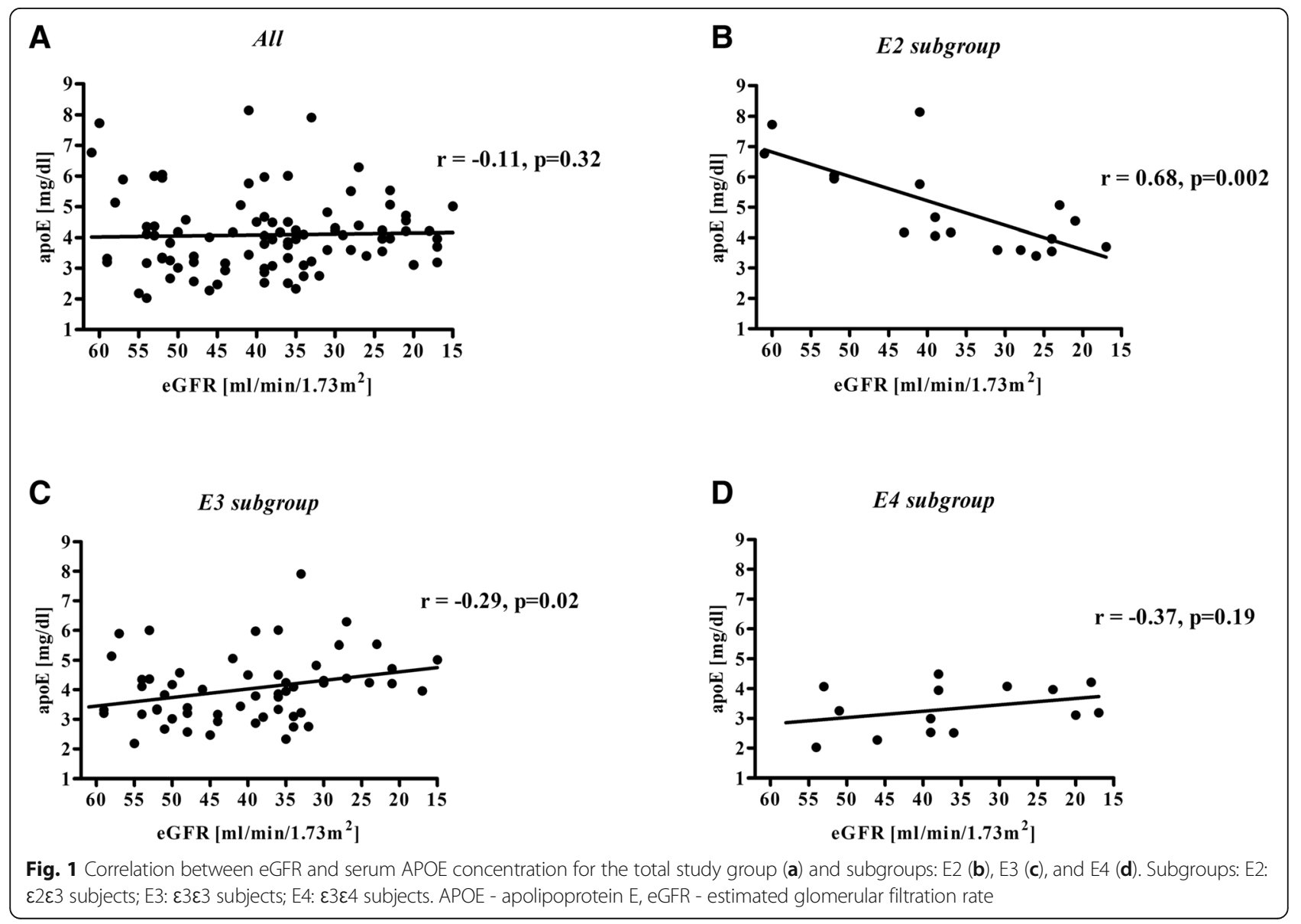


Table 4 Univariate correlation between eGFR CKD-EPI and APOE, APOAI, and APOB concentrations, and apolipoprotein ratios

\begin{tabular}{|c|c|c|c|c|c|c|c|c|}
\hline \multirow[t]{3}{*}{ Parameter } & \multirow{2}{*}{\multicolumn{2}{|c|}{ Total }} & \multicolumn{6}{|c|}{ APOE subgroup } \\
\hline & & & \multicolumn{2}{|l|}{$\overline{E 2}$} & \multicolumn{2}{|l|}{ E3 } & \multicolumn{2}{|l|}{ E4 } \\
\hline & $\mathrm{R}$ & $p$ & $R$ & $p$ & $\mathrm{R}$ & $p$ & $\mathrm{R}$ & $p$ \\
\hline $\mathrm{apoE}$ & -0.11 & 0.32 & 0.68 & 0.002 & -0.29 & 0.02 & -0.37 & 0.19 \\
\hline apoE-HDL & -0.08 & 0.48 & 0.66 & 0.002 & -0.15 & 0.27 & -0.31 & 0.28 \\
\hline apoE-non-HDL & -0.08 & 0.47 & 0.57 & 0.01 & -0.29 & 0.03 & -0.25 & 0.38 \\
\hline apoE-IDL + LDL & 0.09 & 0.39 & 0.78 & $<0.001$ & -0.18 & 0.19 & -0.18 & 0.54 \\
\hline apoE-VLDL & -0.26 & 0.02 & -0.46 & 0.05 & -0.19 & 0.15 & -0.16 & 0.58 \\
\hline apoAl & 0.10 & 0.34 & 0.64 & 0.004 & -0.11 & 0.42 & 0.28 & 0.33 \\
\hline apoE-HDL/apoAl & -0.06 & 0.61 & 0.53 & 0.03 & -0.11 & 0.43 & -0.41 & 0.14 \\
\hline apoB & -0.20 & 0.06 & 0.05 & 0.83 & -0.25 & 0.06 & -0.38 & 0.18 \\
\hline apoB-IDL + LDL & -0.21 & 0.05 & 0.09 & 0.72 & -0.28 & 0.04 & -0.44 & 0.12 \\
\hline apoB-VLDL & -0.03 & 0.81 & -0.10 & 0.69 & 0.07 & 0.61 & 0.13 & 0.67 \\
\hline apoE/apoB in non-HDL & 0.15 & 0.18 & 0.56 & 0.01 & -0.10 & 0.46 & 0.01 & 0.97 \\
\hline apoE/apoB in IDL + LDL & 0.24 & 0.03 & 0.71 & $<0.001$ & 0.02 & 0.89 & 0.07 & 0.82 \\
\hline apoE/apoB in VLDL & -0.26 & 0.02 & -0.34 & 0.17 & -0.26 & 0.07 & -0.17 & 0.56 \\
\hline
\end{tabular}

APOE - apolipoprotein E, APOAI - apolipoprotein Al, APOB - apolipoprotein B, HDL - high density lipoprotein, VLDL - very low-density lipoprotein, IDL -

intermediate density lipoprotein, LDL - low density lipoprotein

Subgroups: E2: $\varepsilon 2 \varepsilon 3$ subjects; E3: $\varepsilon 3 \varepsilon 3$ subjects; E4: $\varepsilon 3 \varepsilon 4$ subjects

APOE level was higher in the E2 subgroup $(p<0.001)$, whereas in the G4 CKD stage it was increased in the E3 subgroup $(p<0.05)$ compared to other subgroups (Fig. 3).

\section{Discussion}

Our findings point to the association of $A P O E$ genotype with APOE concentration and its distribution between lipoproteins in CKD patients depending on kidney function.

Differences in APOE level according to APOE isoform were proven in previous studies, in both non-CKD and CKD patients $[9,22,23,27]$. In accordance with other researchers we observed that APOE level was higher for

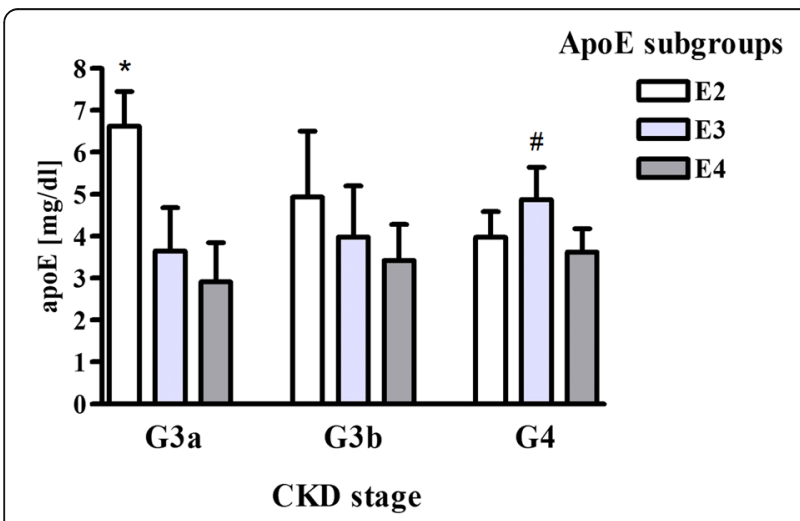

Fig. 2 APOE serum level in CKD patients depending on APOE genotype and CKD stage. Subgroups: E2: $\varepsilon 2 \varepsilon 3$ subjects; E3: $\varepsilon 3 \varepsilon 3$ subjects; E4: $\varepsilon 3 \varepsilon 4$ subjects. Data are expressed as mean \pm SD. ${ }^{*} p<0.001$ vs. E3 and E4 subgroups (G3a stage), $\# p<0.05$ vs. E2 and E4 subgroups (G4 stage). APOE - apolipoprotein E, CKD - chronic kidney disease the E2 subgroup in comparison with the E3 and E4 subgroups [27].

In our own and previous research, it was also observed that APOE concentration did not change with eGFR value decline in CKD patients [6, 35]. However, after adjusting the data for APOE isoform we have established that there is a correlation between APOE level and eGFR; in the $\varepsilon 2$ allele carriers there was a decrease of APOE concentration with the decline of eGFR, while for the E3 subgroup there was a significant increase of APOE level. In the $\varepsilon 4$ allele carriers, there was tendency of an increase in APOE level with eGFR drop, but the statistical significance was not reached. Thus we proved that there is an association between $A P O E$ gene polymorphism and APOE concentration in patients with CKD, during progression of renal dysfunction. To the best of our knowledge this is the first study presenting such a relationship.

For the E2 subgroup, higher APOE concentration in comparison to the E3 and E4 subgroups was observed in the G3a CKD stage. The high APOE level in the $\varepsilon 2$ allele carriers is considered to be a beneficial mechanism, compensating for the decreased affinity of APOE2 to hepatic receptors [36]. Nonetheless, it should be noted that for the $\varepsilon 2$ allele carriers in the G4 CKD stage, the APOE level was significantly lower in comparison with the G3a CKD stage patients, and it was similar or even lower in comparison to the E3 and E4 subgroups (Fig. 3). The mechanism of such a phenomenon should become a subject of further research. 


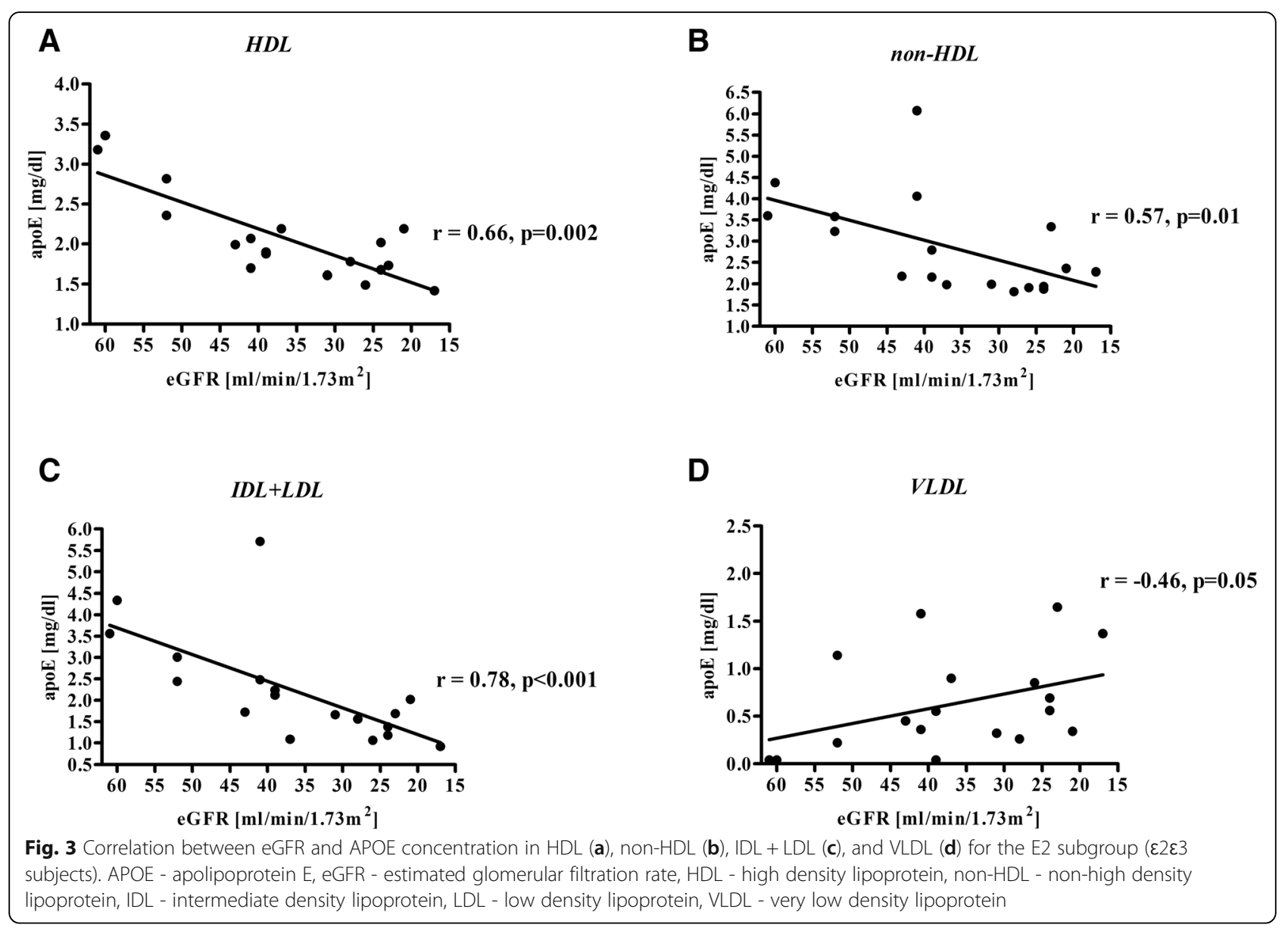

To explain the reason of the correlations observed between APOE level and eGFR we analyzed the APOE level and calculated the APOE/APOAI and APOE/ $\mathrm{APOB}$ ratios for individual lipoprotein fractions.

For the E2 subgroup, we observed a correlation between APOE levels and eGFR for all lipoprotein fractions. For IDL + LDL particles, APOE lowered with a decrease of eGFR. However, APOB - the quantitative marker of IDL and LDL particles in serum, did not decrease, thus we might conclude that the $\varepsilon 2$ carriers in the G4 CKD stage had lower APOE level in IDL + LDL particles, in comparison to the G3a CKD stage patients, but the amount of these lipoproteins was constant along with the CKD progression. [37] (Table 4). The lower content of APOE in IDL + LDL can lead to suppression of the hepatic uptake of these lipoproteins since APOE is a ligand for hepatic LDLR and LRP receptors [38]. Thus, these disturbances could be related to prolonged IDL and LDL clearance from the circulation and their prolonged retention in the arteries walls, which predisposes to atherosclerotic plaques formation [10].

Conversely, the APOE level in VLDL particles increased with progression of renal dysfunction, which may lead to their delayed metabolism and, in a consequence, to hypertriglyceridemia since APOE inhibits lipoprotein lipase activity [39].

In HDL, both the qualitative and quantitative differences in the composition of particles were noticeable in the E2 subgroup for patients with various stages of renal dysfunction. The observed decrease of APOE and APOAI levels in these particles, together with a drop of APOE-HDL/APOAI ratio with the decline of eGFR, may suggest that the number of HDL particles in these patients diminished with the renal dysfuntion, especially those containing APOE. Such HDL disturbances could be related to accelerated atherosclerosis development and higher risk of incident CVD, since it has been proven that the lower amount of HDL as well as lower APOE content in HDL impair all steps of RCT [1, 38-40]. This is due to the fact that APOE participates in cholesterol uptake from the cells in the interaction with the ATP-binding cassette transporter (ABCG1). Moreover, it is an activator of acyltransferase lecithin:cholesterol (LCAT), which esterifies cholesterol in HDL and contributes in cholesteryl esters transmission from HDL to the hepatocytes via Scavenger receptors class B type 1 (SR-B1) and LDL receptors $[13,41]$. In addition, HDL containing APOE are a reservoir of APOE for TG-rich lipoproteins. In the circulation, APOE is transferred from HDL to the IDL 
and LDL particles, allowing for their hepatic clearance [38]. Thus, it can be concluded that a decrease in APOE serum level and its redistribution among lipoprotein classes in $\varepsilon 2$ carriers observed with eGFR decline can promote the progression of lipid disturbances and accelerate atherosclerosis development.

Contrary to the E2 subgroup, for the E3 subgroup APOE-non-HDL level (especially IDL + LDL) increased along with deterioration of renal function. We could suppose that $\varepsilon 3$ homozygotes with more advanced kidney failure accumulated IDL + LDL particles in the plasma since an increase in APOB-(IDL + LDL) concentration with a decline of eGFR has been observed and the ratio of APOE/APOB in IDL + LDL remained constant. Such a phenomenon can be considered as one of the factors accelerating atherosclerosis development, since it has been shown in both non-CKD and CKD patients that APOE level in APOB-containing lipoproteins positively associates with incident CVD risk [22, 42]. Corsetti $J$ et al. showed that high APOE level positively correlated with incident CVD but it referred only to APOE in APOB-containing lipoproteins [22]. Barbagallo $\mathrm{CM}$ et al. proved in a group of males with premature CVD that accumulation of TG-rich lipoproteins with elevated APOE content may comprise an additive factor potentially promoting and initiating the atherosclerotic process [25]. Van Vliet $\mathrm{P}$ et al. showed that in old aged patients high plasma APOE level was associated with a higher risk of stroke [21]. Thus, it can be concluded that for the patients from the E3 subgroup, the increase in APOE level and its contents in lipoproteins with CKD progression are also unfavourable, despite their being different than for the $\varepsilon 2$ carriers.

We did not observe a statistically significant relationship between APOE level and CKD progression for the patients from the E4 subgroup, nevertheless there was a tendency of increase of APOE level with eGFR decline, similar to the E3 subgroup. The lack of statistically significant relationship could be related to the small number of participants in this subgroup, resulting from the low prevalence of the $\varepsilon 4$ allele in the study group. The relatively small number of participants in our research was also due to the many exclusion criteria. However, we could eliminate the possible influence of other chronic illnesses, e.g. diabetes or treatment on APOE level and other lipid and apolipoprotein parameters. We also excluded metabolic syndrome and malnutrition in all of the examined subgroups, which could potentially influence lipid and lipoprotein metabolism. Nevertheless, it should be emphasised that the frequency of $A P O E$ gene polymorphism in our study group was comparable to APOE isoform occurrence in other studies in CKD and general populations [6, 14, 43].

\section{Conclusions}

In summary, our results support earlier reports about the relationship between $A P O E$ gene polymorphism and APOE concentration. However, we have shown for the first time that in CKD patients, the APOE level and its distribution between lipoprotein classes is associated not only with $A P O E$ gene polymorphism but also with kidney function. Therefore, it can be concluded that lipid and lipoprotein disorders in CKD should be analyzed considering $A P O E$ gene polymorphism. Further observation is crucial to explain the role of the association between $A P O E$ gene polymorphism and lipid disturbances in CVD development in CKD, as well as to establish if there is a link between lipid disturbances for particular $\varepsilon$ allele carriers and increased rate of CKD progression.

\section{Abbreviations \\ APO: apolipoprotein; BMI: body mass index; BP: blood pressure; CKD: chronic kidney disease; CKD-EPI: chronic kidney disease epidemiology collaboration; CM: chylomicrons; CVD: cardiovascular disease; eGFR: estimated glomerular filtration rate; HDL: high density lipoprotein; HTG: hypertriglyceridemia; IDL: intermediate density lipoprotein; LDL: low density lipoprotein; LDLR: Iow density lipoprotein receptor; LPR: LDL receptor-related protein; Non- HDL: non-high density lipoprotein (IDL + LDL); PCR: polymerase chain reaction; $\mathrm{RCT}$ : reverse cholesterol transport; TC: total cholesterol; TG: triglyceride; TP: total protein; VLDL: very low-density lipoprotein; WHR: waist-to-hip ratio \\ Acknowledgements \\ not applicable. \\ Funding \\ This work was supported by the Medical University of Gdansk grants nos. ST 02-0004/07/122, ST 02-0125/07/524, and MN 01-0219/08/524. \\ Availability of data and materials \\ The datasets analysed during the current study are available from the corresponding author on reasonable request. \\ Authors' contributions \\ $M C$ and EK recruited CKD patients. AC and BK-S designed the study. MC col- lected the clinical data, which was supervised by EK and AD-Ś. AĆ, EW, MS-B, RK performed the laboratory analysis. MC, AC, AK performed the data ana- lysis. MC drafted the manuscript. MC and AĆ made the graphics. EK, BK-S, MJ, AC revised the manuscript. All authors read and approved the final manuscript.}

\section{Ethics approval and consent to participate}

The Independent Bioethics Commission for Research of the Medical University of Gdańsk (Poland) approved the study (NKBBN/541-256/2017).

\section{Consent for publication}

not applicable.

\section{Competing interests}

The authors declare that they have no competing interests.

\section{Publisher's Note}

Springer Nature remains neutral with regard to jurisdictional claims in published maps and institutional affiliations.

\section{Author details}

${ }^{1}$ Clinic \& Chair of Nephrology, Transplantology and Internal Medicine, Medical University of Gdańsk, Dębinki 7, 80-211 Gdańsk, Poland. ²Department of Clinical Chemistry, Medical University of Gdańsk, Dębinki 7, 80-211 Gdańsk, Poland. ${ }^{3}$ Department of Molecular Medicine, Medical University of Gdańsk, 
Dębinki 7, 80-211 Gdańsk, Poland. ${ }^{4}$ Department of Therapy Monitoring and Pharmacogenetics, Medical University of Gdańsk, Dębinki 7, 80-211 Gdańsk, Poland.

Received: 12 October 2018 Accepted: 1 March 2019

Published online: 09 March 2019

\section{References}

1. Stevens PE, Levin A. Evaluation and management of chronic kidney disease: synopsis of the kidney disease: improving global outcomes 2012 clinical practice guideline. Ann Intern Med United States; 2013;158(11):825-830.

2. Visconti L, Benvenga S, Lacquaniti A, Cernaro V, Bruzzese A, Conti G, et al. Lipid disorders in patients with renal failure: role in cardiovascular events and progression of chronic kidney disease. J Clin Transl Endocrinol Netherlands; 2016;6:8-14.

3. Tsimihodimos V, Dounousi E, Siamopoulos KC. Dyslipidemia in chronic kidney disease: an approach to pathogenesis and treatment. Am J Nephrol Switzerland; 2008;28(6):958-973.

4. Rutkowski B, Łososowska R, Krol E, Kisielnicka E, Zdrojewski Z, Szołkiewicz M, et al. Patomechanizm hiperlipoproteinemii w przewlekłej niewydolności nerek. Pol Merkur Lek. 2003;15:322-5.

5. Wroblewska M, Krol E. Dyslipoproteinemie w chorobach nerek. Pol Tyg Lek (Wars). 1993:48:495-7.

6. Krol E, Rutkowski B, Wroblewska M, Badzio T. Classification of lipid disorders in chronic hemodialyzed patients. Miner Electrolyte Metab Switzerland; 1996:22(1-3):13-15.

7. Cwiklinska A, Cackowska M, Wieczorek E, Krol E, Kowalski R, Kuchta A, et al. Progression of chronic kidney disease affects HDL impact on lipoprotein lipase (LPL)-mediated VLDL lipolysis efficiency. Kidney Blood Press Res Switzerland; 2018;43(3):970-978.

8. Attman PO, Alaupovic P, Gustafson A. Serum apolipoprotein profile of patients with chronic renal failure. Kidney Int United States; 1987;32(3):368-375.

9. Davignon J, Gregg RE, Sing CF. Apolipoprotein E polymorphism and atherosclerosis. Arteriosclerosis United States; 1988;8(1):1-21.

10. Heeren J, Beisiegel U, Grewal T. Apolipoprotein E recycling: implications for dyslipidemia and atherosclerosis. Arterioscler Thromb Vasc Biol. United States; 2006;26(3):442-448.

11. Mahley RW. Apolipoprotein E: cholesterol transport protein with expanding role in cell biology. Science United States; 1988 Apr;240(4852):622-630.

12. Meir KS, Leitersdorf E. Atherosclerosis in the apolipoprotein-E-deficient mouse: a decade of progress. Arterioscler Thromb Vasc Biol United States; 2004;24(6):1006-1014.

13. Greenow K, Pearce NJ, Ramji DP. The key role of apolipoprotein E in atherosclerosis. J Mol med (Berl) Germany; 2005;83(5):329-342.

14. Bahri R, Esteban E, Moral P, Hassine M, Ben Hamda K, Chaabani H. Apolipoprotein gene polymorphisms and plasma levels in healthy Tunisians and patients with coronary artery disease. Lipids Health Dis. England; 2008;7:46.

15. Lin CY, Duan H, Mazzone T. Apolipoprotein E-dependent cholesterol efflux from macrophages: kinetic study and divergent mechanisms for endogenous versus exogenous apolipoprotein E. J Lipid Res. United States; 1999;40(9):1618-1627.

16. Mahley RW, Apolipoprotein E: From cardiovascular disease to neurodegenerative disorders. J Mol med (Berl). Germany; 2016;94(7):739-746.

17. Nguyen D, Dhanasekaran P, Nickel M, Nakatani R, Saito H, Phillips MC, et al. Molecular basis for the differences in lipid and lipoprotein binding properties of human apolipoproteins E3 and E4. Biochemistry United States; 2010;49(51):10881-10889.

18. Vincent-Viry M, Schiele F, Gueguen R, Bohnet K, Visvikis S, Siest G. Biological variations and genetic reference values for apolipoprotein $\mathrm{E}$ serum concentrations: results from the STANISLAS cohort study. Clin Chem. United States; 1998;44(5):957-965.

19. Morton AM, Koch M, Mendivil CO, Furtado JD, Tjonneland A, Overvad K, et al. Apolipoproteins $\mathrm{E}$ and $\mathrm{CIII}$ interact to regulate $\mathrm{HDL}$ metabolism and coronary heart disease risk. JCl insight. United States; 2018;3(4).

20. Reilly M, Rader DJ. Apolipoprotein E and coronary disease: a puzzling paradox. PLoS Med United States; 2006 Jun;3(6):e258.

21. van Vliet P, Mooijaart SP, de Craen AJM, Rensen PCN, van Heemst D, Westendorp RGJ. Plasma levels of apolipoprotein $E$ and risk of stroke in old age. Ann N Y Acad Sci. United States; 2007;1100:140-147.

22. Corsetti JP, Gansevoort RT, Bakker SJL, Dullaart RPF. Apolipoprotein E levels and apolipoprotein E genotypes in incident cardiovascular disease risk in subjects of the prevention of renal and vascular end-stage disease study. J Clin Lipidol United States; 2016;10(4):842-850.

23. Xue C, Nie W, Tang D, Yi L, Mei C. Apolipoprotein E gene variants on the risk of end stage renal disease. PLoS One. United States; 2013;8(12):e83367.

24. Ferreira CN, Carvalho MG, Fernandes APSM, Lima LM, Loures-Valle AA, Dantas J, et al. Comparative study of apolipoprotein-E polymorphism and plasma lipid levels in dyslipidemic and asymptomatic subjects, and their implication in cardio/cerebro-vascular disorders. Neurochem Int England; 2010:56(1):177-182.

25. Barbagallo CM, Rizzo M, Noto D, Frasheri A, Pernice $V$, Rubino A, et al. Accumulation of apoE-enriched triglyceride-rich lipoproteins in patients with coronary artery disease. Metabolism United States; 2006;55(5):662-668.

26. Cwiklinska A, Strzelecki A, Kortas-Stempak B, Zdrojewski Z, Wroblewska M. [ApoE-containing HDL and the development of atherosclerosis]. Postepy Hig Med Dosw (Online). Poland; 2015;69:1-9.

27. Wang Y, Wang N, Lu Y, Yu Q, Zhou L, Xu Q. Detection of apolipoprotein E gene polymorphism and blood lipid level in hemodialysis patients. J Clin Med Res Canada; 2017;9(8):695-700.

28. Dong C-Q, Luo Y-G, Dong K, Chen C, Liu Q, Yang T-Q. Relationship between apolipoprotein $\mathrm{E}$ gene polymorphism with triglyceride level in patients with renal diseases. Ren Fail England; 2013;35(10):1449-1454.

29. McEneny J, Loughrey CM, McNamee PT, Trimble ER, Young IS. Susceptibility of VLDL to oxidation in patients on regular haemodialysis. Atherosclerosis. Ireland: 1997:129(2):215-220.

30. Wroblewska M, Kortas-Stempak B, Szutowicz A, Badzio T. Phospholipids mediated conversion of HDLs generates specific apoA-II pre-beta mobility particles. J Lipid Res United States; 2009;50(4):667-675.

31. Kukita H, Hamada M, Hiwada K, Kokubu T. Clinical significance of measurements of serum apolipoprotein A-l, A-II and B in hypertriglyceridemic male patients with and without coronary artery disease. Atherosclerosis Ireland; 1985;55(2):143-149.

32. Marz W, Feussner G, Siekmeier R, Donnerhak B, Schaaf L, Ruzicka V, et al. Apolipoprotein $\mathrm{E}$ to $\mathrm{B}$ ratio: a marker for type III hyperlipoproteinaemia. Eur J Clin Chem Clin Biochem Germany; 1993;31(11):743-747.

33. Dodder NG, Tai SS-C, Sniegoski LT, Zhang NF, Welch MJ. Certification of creatinine in a human serum reference material by GC-MS and LC-MS. Clin Chem United States; 2007:53(9):1694-1699.

34. Chen $\mathrm{C}-\mathrm{H}$. Development of a melting curve-based allele-specific PCR of apolipoprotein E (APOE) genotyping method for genomic DNA, Guthrie blood spot, and whole blood. PLoS One United States; 2016;11(4):e0153593.

35. Attman PO, Alaupovic P, Tavella M, Knight-Gibson C. Abnormal lipid and apolipoprotein composition of major lipoprotein density classes in patients with chronic renal failure. Nephrol Dial Transplant England; 1996;11(1):63-69.

36. Han $S, X u$ Y, Gao M, Wang Y, Wang J, Liu Y, et al. Serum apolipoprotein E concentration and polymorphism influence serum lipid levels in Chinese Shandong Han population. Medicine (Baltimore). United States; 2016;95(50): e5639.

37. Cho D-S, Woo S, Kim S, Byrne CD, Kong J-H, Sung K-C. Estimation of plasma apolipoprotein B concentration using routinely measured lipid biochemical tests in apparently healthy Asian adults. Cardiovasc Diabetol. England; 2012;11:55.

38. Phillips MC. Apolipoprotein E isoforms and lipoprotein metabolism. IUBMB Life England; 2014;66(9):616-623.

39. Rensen PC, van Berkel TJ. Apolipoprotein E effectively inhibits lipoprotein lipase-mediated lipolysis of chylomicron-like triglyceride-rich lipid emulsion in vitro and in vivo. J Biol Chem United States; 1996 Jun:271(25):1479114799.

40. Wilson HM, Patel JC, Russell D, Skinner ER. Alterations in the concentration of an apolipoprotein E-containing subfraction of plasma high density lipoprotein in coronary heart disease. Clin Chim Acta Netherlands; 1993 Nov;220(2):175-187.

41. Krimbou L, Denis M, Haidar B, Carrier M, Marcil M, Genest JJ. Molecular interactions between apoE and ABCA1: impact on apoE lipidation. J Lipid Res. United States; 2004;45(5):839-848.

42. Mendivil CO, Rimm EB, Furtado J, Sacks FM. Apolipoprotein E in VLDL and LDL with apolipoprotein C-III is associated with a lower risk of coronary heart disease. J Am Heart Assoc England; 2013;2(3):e000130.

43. Liberopoulos E, Siamopoulos K, Elisaf M. Apolipoprotein E and renal disease. Am J Kidney Dis United States; 2004;43(2):223-233. 